\title{
Astrometric reduction of Cassini ISS images of the Saturnian satellites Mimas and Enceladus ${ }^{\star}$
}

\author{
R. Tajeddine ${ }^{1,3}$, N. J. Cooper ${ }^{1,2}$, V. Lainey ${ }^{1}$, S. Charnoz ${ }^{3}$, and C. D. Murray ${ }^{2}$ \\ 1 IMCCE, Observatoire de Paris, UMR 8028 du CNRS, UPMC, Université de Lille 1, 77 av. Denfert-Rochereau, 75014 Paris, France \\ e-mail: tajeddine@imcce.fr \\ 2 Astronomy Unit, School of Physics and Astronomy, Queen Mary University of London, Mile End Road, London E1 4NS, UK \\ ${ }^{3}$ Laboratoire AIM, UMR 7158, Université Paris Diderot - CEA IRFU - CNRS, Centre de l'Orme les Merisiers, 91191 Gif-sur-Yvette \\ Cedex, France
}

Received 2 December 2012 / Accepted 6 February 2013

\begin{abstract}
Aims. We provide astrometric observations of two of Saturn's main satellites, Mimas and Enceladus, using high resolution Cassini ISS Narrow Angle Camera images.

Methods. We developed a simplified astrometric reduction model for Cassini ISS images as an alternative to the one proposed by the Jet Propulsion Labratory (JPL). The particular advantage of the new model is that it is easily invertible, with only marginal loss in accuracy. We also describe our new limb detection and fitting technique.

Results. We provide a total of 1790 Cassini-centred astrometric observations of Mimas and Enceladus, in right ascension $(\alpha)$ and declination $(\delta)$ in the International Celestial Reference Frame (ICRF). Mean residuals compared to JPL ephemerides SAT317 and SAT351 of about one kilometre for Mimas and few hundreds of metres for Enceladus were obtained, in $\alpha \cos \delta$ and $\delta$, with a standard deviation of a few kilometres for both satellites. A frequency analysis of the residuals revealed some periodic variability in the right ascension for Mimas. An additional analysis of Mimas' mean longitude suggests that some short-period terms are missing in the TASS orbital model.
\end{abstract}

Key words. astrometry - planets and satellites: individual: Mimas - planets and satellites: individual: Enceladus methods: observational

\section{Introduction}

Over the past few decades spacecraft imaging has increasingly entered the field of astrometry and images from spacecraft missions are now routinely used to measure the astrometric positions of planetary satellites in order to study their orbital motion. For instance, Jacobson (1991) reduced observations of the Neptunian satellites, Triton and Nereid, from Voyager 2 optical images. Jacobson (1992) also reduced observations of the major Uranian satellites using images taken by the same spacecraft, while astrometry of the Martian satellites, Phobos and Deimos, has been performed using images from the Mars Express spacecraft (Willner 2008). Hubble Space telescope (HST) observations of the satellites of Saturn have been also been reduced astrometrically by French (2006), and Cooper et al. (2006) reduced Cassini Imaging Science Subsystem (ISS) observations of the inner Jovian satellites, Amalthea and Thebe. All these examples have demonstrated the high accuracy that can be obtained from the astrometric reduction of spacecraft images compared to ground-based imaging, and spacecraft astrometry has now proven its importance in obtaining accurate planetary satellite ephemerides.

In this paper we describe the astrometric reduction of Cassini ISS narrow angle camera (NAC) observations of two of Saturn's main satellites, Mimas and Enceladus, two satellites whose dynamical and physical evolution are now considered to be of

* Full Table 4 is only available at the CDS

via anonymous ftp to cdsarc.u-strasbg. fr $(130.79 .128 .5)$ or via http: //cdsarc.u-strasbg.fr/viz-bin/qcat?J/A+A/551/A129 crucial importance in understanding the evolution of the Saturn system as a whole. In the case of Enceladus, geysers were discovered by the Cassini spacecraft in the south polar region (Porco et al. 2006) revealing the existence of liquid water beneath the surface, while an orbital acceleration has been reported by Lainey et al. (2012) for Mimas, which could have important consequences for the dynamical evolution of Saturn's rings (Noyelles et al. 2012).

The Cassini spacecraft commenced its prime mission in 2004, following Saturn-orbit-insertion in July of that year. Up until February 2011, the Cassini ISS NAC had produced approximately 9000 high resolution optical images of the Saturnian satellites Mimas and Enceladus. Of these we have found 1790 to be suitable for astrometry. For the remainder, astrometric reduction has not been possible either because suitable background stars necessary for astrometric reduction were not detectable, or the satellite was covering the entire field-of-view, or Saturn was observed in the background (we describe these issues in more detail in the sections that follow). In Sect. 2 we introduce our new astrometric reduction model, based on an approach more generally used for ground-based observations, and compare it to the Cassini ISS model proposed by Owen (2003). In the same section, we describe our satellite limb-measuring technique, inspired by Mallama et al. (2004), and our method for fitting an elliptical model of the satellite's shape to the measured limb. We also evaluate the importance of image distortion in limb-fitting. Astrometric observations and statistical analyses are given in Sect. 3, while further discussion and conclusions follow in the final section 


\section{Astrometry}

Astrometric calibration of a given image involves firstly correcting the camera's orientation and pointing direction, based on the position of background reference stars. In this section we describe our new model to convert a position given in right ascension and declination coordinates (in radians) to sample and line coordinates (in pixels) in a Cassini NAC image.

The images used in this work were downloaded from the Planetary Data System (PDS) website ${ }^{1}$. After photometric calibration using the CISSCAL software package (West et al. 2010), the right ascension and declination $\left(\alpha_{\mathrm{c}}, \delta_{\mathrm{c}}\right)$ of the optical axis of the NAC for each image was determined at the time of observation, using the mid-times extracted from the image headers and the appropriate SPICE kernels available within the SPICE library $^{2}$ (Acton 1996). Given the camera orientation and its fieldof-view, we then used the UCAC2 star catalogue (Zacharias et al. 2004) to locate reference stars within the field-of-view. This catalogue provides star positions in terms of their right ascension and declination $\left(\alpha_{*}, \delta_{*}\right)$ in the International Celestial Reference Frame (ICRF).

\subsection{Astrometric model}

Our astrometric reduction algorithm proceeds as follows: a star's coordinates $\left(\alpha_{*}, \delta_{*}\right)$ are first corrected for proper motion, aberration, and relativistic effects, and the observer's location translated from solar system barycentric coordinates to the Cassini spacecraft frame (Kaplan et al. 1989). A gnomonic projection is then used to convert the corrected star coordinates from the celestial sphere (with a field of view of $\approx 0.35^{\circ} \times 0.35^{\circ}$ ) to coordinates $(X, Y)$ in the tangential plane of the camera's CCD, using Eqs. (1) and (2) (see also Eichhorn 1974 for further details). After a further conversion from radians to arcsec, we then finally convert to sample and line $(s, l)$ in pixels, using Eq. (3) below:

$$
\begin{aligned}
& X=\frac{\cos \delta_{*} \sin \left(\alpha_{*}-\alpha_{\mathrm{c}}\right)}{\sin \delta_{*} \sin \delta_{\mathrm{c}}+\cos \delta_{*} \cos \delta_{\mathrm{c}} \cos \left(\alpha_{*}-\alpha_{\mathrm{c}}\right)} \\
& Y=\frac{\sin \delta_{*} \cos \delta_{\mathrm{c}}-\cos \delta_{*} \sin \delta_{\mathrm{c}} \cos \left(\alpha_{*}-\alpha_{\mathrm{c}}\right)}{\sin \delta_{*} \sin \delta_{\mathrm{c}}+\cos \delta_{*} \cos \delta_{\mathrm{c}} \cos \left(\alpha_{*}-\alpha_{\mathrm{c}}\right)} \\
& \left(\begin{array}{l}
s \\
l
\end{array}\right)=\left(\begin{array}{cc}
-1 / \rho_{s} & 0 \\
0 & 1 / \rho_{l}
\end{array}\right)\left(\begin{array}{cc}
\cos (\theta) & -\sin (\theta) \\
\sin (\theta) & \cos (\theta)
\end{array}\right)\left(\begin{array}{l}
X \\
Y
\end{array}\right)+\left(\begin{array}{c}
s_{0} \\
l_{0}
\end{array}\right) .
\end{aligned}
$$

In Eq. (3), $\rho_{s}$ and $\rho_{l}$ are the scale factors for sample and line, respectively, measured in arcsec/pixel. Note that at this stage, we introduce two scale factors, thus assuming a rectangular pixel shape.

Since the algorithm has been coded following the conventions used in the IDL programming language, the origin of the sample and line coordinate system is at the centre of the bottom left pixel, with sample increasing to the right and line increasing upward, when the image is displayed in its line-reversed orientation. The spacecraft's "north" is aligned with its $+X$ axis in the increasing line direction and its "east" is aligned with the $-Z$ axis in the decreasing sample direction, hence the "-" sign before $\rho_{s}$ (see Porco et al. (2004) for further details on Cassini's ISS reference frame conventions). Angle $\theta$ denotes the angle between the projection of the ICRF $Z$ axis on the spacecraft's $(X Z)$ plane and the spacecraft's $Z$ axis, commonly referred to as the twist angle.

\footnotetext{
1 Planetary Data System (PDS) URL: http://pds.nasa.gov/

2 SPICE library's URL: http://naif.jpl .nasa.gov/naif/
}

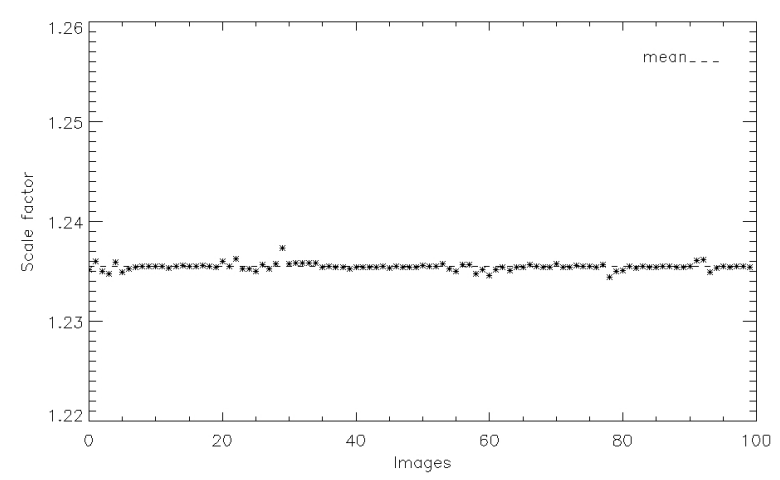

Fig. 1. Derived scale factors (considering a square pixel shape) versus image sequence number. The dashed line is the mean value of $1.2354 \mathrm{arcsec} / \mathrm{pixel}$.

\begin{tabular}{|c|c|c|c|c|c|}
\hline & $\begin{array}{c}\rho_{s} \\
(\operatorname{arcsec} / \text { pixel })\end{array}$ & $\begin{array}{c}\rho_{l} \\
(\operatorname{arcsec} / \text { pixel })\end{array}$ & $\begin{array}{c}\sigma\left(\rho_{s}\right) \\
(\operatorname{arcsec} / \text { pixel })\end{array}$ & $\begin{array}{c}\sigma\left(\rho_{l}\right) \\
(\operatorname{arcsec} / \text { pixel })\end{array}$ & $\begin{array}{c}\langle\mathrm{rms}\rangle \\
\text { (pixels) }\end{array}$ \\
\hline$\rho_{s} \neq \rho_{l}$ & 1.2355 & 1.2354 & $10^{-4}$ & $10^{-4}$ & 0.33 \\
\hline$\rho_{s}=\rho_{l}$ & \multicolumn{2}{|c|}{1.2354} & \multicolumn{2}{|c|}{$3 \times 10^{-4}$} & 0.35 \\
\hline
\end{tabular}

Table 1. Values of scale factors in arcsec/pixel for rectangular pixels $\left(\rho_{s} \neq \rho_{l}\right)$ and square pixels $\left(\rho_{s}=\rho_{l}\right)$, respectively.

Finally, $s_{0}$ and $l_{0}$ represent the necessary translations (both equal to 511.5 for $1024 \times 1024$ pixel images) for image centring.

For full astrometric calibration, five parameters should ideally be fitted for each image: $\rho_{s}, \rho_{l}, \alpha_{\mathrm{c}}, \delta_{\mathrm{c}}$ and $\theta$. The last three parameters vary from image to image. However the first two are properties of the camera, and hence common to each image, and can therefore be fixed in order to reduce the observational errors. We therefore calibrated the first two parameters by selecting 100 images from two series of images of star clusters (image series N1580739191 to N1580748341 and N1601334486 to N1601342286, respectively) with a mean number of 225 stars detected per image. Initially, calibration was performed assuming $\rho_{s} \neq \rho_{l}$. The same calibration was then repeated with $\rho_{s}=\rho_{l}$ (Fig. 1). In Fig. 1 we see that the value of the scale factor remains stable and image-independent. Table 1 shows the values of scale factors used in both cases. From this table we conclude that considering a square shape of pixel is a reasonable approximation and we therefore chose $\rho_{s}=\rho_{l}=1.2354 \mathrm{arcsec} / \mathrm{pixel}$, reducing the number of parameters to be fitted to three. Since each star provides information for two fitted parameters, we need a minimum of two stars to correct the camera's pointing vector and twist angle. Thus, all observations that have been published in this paper contain at least two detected stars per image.

Having measured the satellite's imaged position in sample and line $(s, l)$, it is then necessary to be able to obtain the equivalent position in $\left(\alpha_{s}, \delta_{s}\right)$. Equation (4) is provided for this purpose. This equation is the inversion of Eq. (3), and converts a position from $(s, l)$ to $(X, Y)$. Equations (5) and (6) can then be used to transform the resulting position in $(X, Y)$ to $\left(\alpha_{s}, \delta_{s}\right)$.

$$
\begin{aligned}
& \left(\begin{array}{l}
X \\
Y
\end{array}\right)=\left(\begin{array}{cc}
-\rho_{s} & 0 \\
0 & \rho_{l}
\end{array}\right)\left(\begin{array}{cc}
\cos (\theta) & -\sin (\theta) \\
\sin (\theta) & \cos (\theta)
\end{array}\right)\left(\begin{array}{l}
s-s_{0} \\
l-l_{0}
\end{array}\right) \\
& \alpha_{s}=\alpha_{\mathrm{c}}+\arctan \left(\frac{X}{\cos \delta_{\mathrm{c}}-Y \sin \delta_{\mathrm{c}}}\right) \\
& \delta_{s}=\arctan \left(\frac{\sin \delta_{\mathrm{c}}+Y \cos \delta_{\mathrm{c}}}{\sqrt{X^{2}+\left(\cos \delta_{\mathrm{c}}-Y \sin \delta_{\mathrm{c}}\right)^{2}}}\right)
\end{aligned}
$$


Table 2. Comparison of rms residuals (pixel) between this paper's model and Owen's model for 50 images, with a mean number of stars per image $=225$.

\begin{tabular}{lcc}
\hline \hline & $\langle$ rms $\rangle$ (pixels) & $\sigma_{\text {rms }}$ (pixels) \\
\hline Present model & 0.32 & 0.027 \\
Owen's model & 0.30 & 0.017 \\
\hline
\end{tabular}

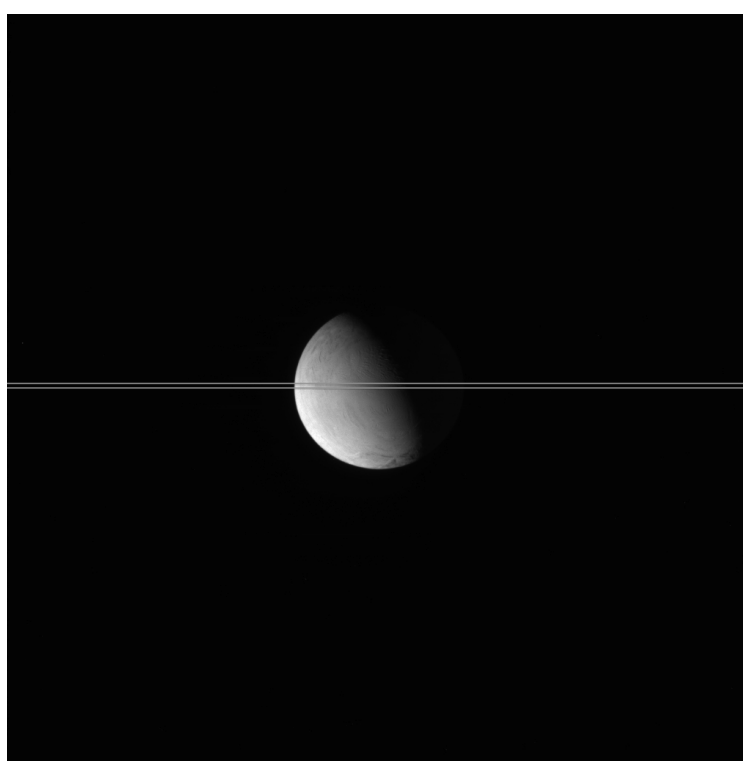

Fig. 2. Image of Enceladus and a line showing the area where the pixel intensity is being studied.

\subsection{Owen's model}

Owen (2003) developed a model for the astrometric reduction of Cassini ISS images, including the effects of camera distortion, in which the properties of the Cassini ISS cameras were determined empirically from images of the open star cluster M 35 (the model is also described in Cooper et al. 2006). In this model, camera distortion is treated via the following equations:

$$
\left(\begin{array}{c}
\Delta x \\
\Delta y
\end{array}\right)=\left(\begin{array}{lll}
x r^{2} & x y & x^{2} \\
y r^{2} & y^{2} & x y
\end{array}\right)\left(\begin{array}{c}
\epsilon_{2} \\
\epsilon_{5} \\
\epsilon_{6}
\end{array}\right)
$$

where $r^{2}=x^{2}+y^{2}, \epsilon_{\mathrm{n}}$ are camera-dependent parameters whose numerical values are given in Owen (2003). Thus, distortion transforms each point $(x, y)$ on the CCD to $(x+\Delta x, y+\Delta y)$, where $\Delta x$ and $\Delta y$ are given by the above expressions. By comparison, in our model (described earlier), distortion is not included in one explicit equation, but instead is partly absorbed during the scalefactor fitting. This accounts for the difference between the scale factor derived in this paper $(\approx 1.2354 \mathrm{arcsec} / \mathrm{pixel})$, and Owen's value $(\approx 1.2357$ arcsec/pixel).

To compare both models, we chose 50 images from the same series used by Owen (2003), selected on the basis of their high star count. Star detection was performed using the IDL-based "FIND" (Stetson 1987) routine, available online from the IDL Astronomy User's Library ${ }^{3}$, detecting a mean number of 225 stars per image. A comparison of the mean postfit rms residuals between the two models (using $\rho_{s}=\rho_{l}=$ $1.2354 \mathrm{arcsec} / \mathrm{pixel}$ ) is shown in Table 2 . It is noteworthy that the

\footnotetext{
3 IDL Astronomy User's Library URL: http://idlastro.gsfc. nasa.gov/
}

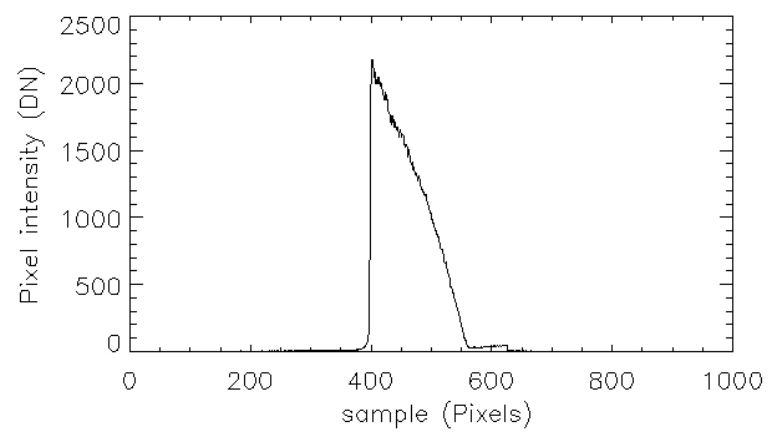

Fig. 3. Pixel intensity (DN) profile of the chosen area in Fig. 2.

satellite resolution depends on its distance to the spacecraft. For Mimas the resolution varies from $40 \mathrm{~km} /$ pixel to $0.5 \mathrm{~km} /$ pixel, and from $35 \mathrm{~km} /$ pixel to $0.5 \mathrm{~km} /$ pixel for Enceladus. Therefore, according to the comparison in Table 2 , a variation of $10^{-2}$ pixels results in a variation in the satellite position of $5 \times 10^{-2}$ to $4 \times 10^{-1}$ kilometres for Mimas and $5 \times 10^{-2}$ to $3.5 \times 10^{-1}$ kilometres for Enceladus. Thus, the new model compares favourably with Owen's model and despite the approximations used, can still provide sufficiently accurate astrometry. On the other hand, although Owen's model is slightly more accurate, the presence of non-linear terms make the inversion of these equations in order to convert a position from (sample, line) to $(\alpha, \delta))$ more problematic, without compromising the observation accuracy. Thus in order to take full advantage of the accuracy obtainable with Owen's model, it is generally desirable to leave the observed positions in (sample, line) format. The benefit of the new model is that it provides the satellite's observed position in $(\alpha, \delta)$ instead of (sample, line), which is the conventional coordinate system for published astrometric observations, without the need for an iterative inversion technique as would be required with Owen's model. Overall therefore, the new model is easy to introduce in any astrometric software, for only a small decrease in accuracy, and on this basis we have decided to proceed with our model for the astrometric reduction described in this work. Later, in Sect. 3 where we analyse the residuals, we show that the camera have been modeled well enough.

\subsection{Satellite position measurement}

Following correction of the camera's orientation and pointing direction, the next step is to find the centre-of-figure of the observed satellite. Since the satellites were fully-resolved in the Cassini ISS NAC images selected for this work, it was necessary to use a limb-measuring and fitting approach to find the centre-of-figure, rather than using a centroiding technique with associated phase correction.

\subsubsection{Limb measurement}

Let us consider an image of Enceladus as shown in Fig. 2. To measure one point (in sample and line) of the satellite's apparent limb, the following steps must be applied:

- First, obtain the horizontal profile of pixel intensity (DN) across the satellite (Fig. 3).

- Second, compute the absolute value of the derivative of the pixel intensity curve (Fig. 4). The horizontal position of the limb corresponds to the horizontal position of the maximum of this curve. An image-dependent threshold must be added 


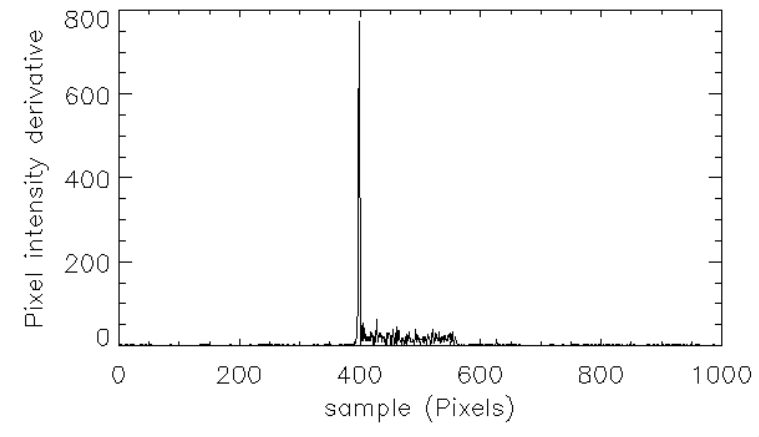

Fig. 4. Absolute value of the derivative of the Pixel intensity curve in Fig. 3. The maximum represents one point of the limb.

when searching for the maximum in order to avoid detecting points from the terminator.

- Finally, repeat the first two steps for all lines and samples to obtain the coordinates of all detectable limb points.

\subsubsection{Ellipse fitting}

Having detected the satellite limb in the image, the next step is to fit a model of the shape of the satellite to the measured limb points. The JPL SPICE library incorporates approximate shape models for all major natural satellites in the form of triaxial ellipsoids, based on the shapes derived by Thomas et al. (2007). For a given satellite, the SPICE ellipsoid was projected on to the image, forming an ellipse which could then be fitted to the measured limb points using a least-squares technique. Such an ellipse can be parameterised in terms of five quantities: the coordinates $X_{\mathrm{c}}$ and $Y_{\mathrm{c}}$, of the centre of the ellipse, the semi-major and semi-minor axes and the angle of orientation of the ellipse $\phi$. However, given that the semi-major and the semi-minor axes are properties of the full ellipsoid, these parameters are known in advance, and hence only the remaining three parameters need to be fitted to the measured limb in order to determine the centre of the ellipse i.e. the centre-of-figure of the satellite.

To evaluate the effects of camera distortion on the satellite centre measurements, limb-fitting was performed twice on 100 randomly-selected images of Mimas and Enceladus (with random resolutions): once with the distortion correction (Eq. (7)) and once without. Table 3 shows the difference in sample and line (in pixels) of the measured satellite's centre of figure. We conclude from these results that the camera distortion correction does not significantly affect the measurement of the satellite's position.

\section{Observations and analysis}

Using the astrometric and limb-fitting techniques described in the previous section, we reduced 870 out of about 2500 available NAC images of Mimas and 920 out of about 5500 of available NAC images of Enceladus. The complete set of reduced observations is available at the CDS. Table 4 shows a small sample of these observations. The variables in this table are described as follows: Image name, Date and exposure mid-time of the image (UTC), Satellite name, Right ascension and Declination of the satellite (in the ICRF), observation uncertainties in Right ascension and Declination, Right ascension and Declination of the camera's pointing vector and the twist angle of the pointing vector ( $\theta$ in Eq. (3)), Sample and Line (in pixels) of the observed
Table 3. Effects of camera distortion on the satellite's measured limb (made on 100 images of Mimas and Enceladus).

\begin{tabular}{lcccc}
\hline \hline & $\begin{array}{c}\left\langle\Delta_{s}\right\rangle \\
\text { (pixels) }\end{array}$ & $\begin{array}{c}\left\langle\Delta_{l}\right\rangle \\
\text { (pixels) }\end{array}$ & $\begin{array}{c}\sigma_{\Delta s} \\
\text { (pixels) }\end{array}$ & $\begin{array}{c}\sigma_{\Delta l} \\
\text { (pixels) }\end{array}$ \\
\hline With minus without distortion correction & -0.011 & -0.022 & 0.032 & 0.033 \\
$\langle\mathrm{O}-\mathrm{C}\rangle$ (without distortion correction) & 0.184 & 0.712 & 1.020 & 0.869 \\
$\langle\mathrm{O}-\mathrm{C}\rangle$ (with distortion correction) & 0.196 & 0.735 & 1.018 & 0.878 \\
\hline
\end{tabular}

Notes. "With distortion" means that the distortion effect was included in limb measurement, "Without distortion" means that no distortion effect added on limb measurement. The first line shows a comparison between satellite positions when adding this effect and removing it. The last two lines show the comparison between the observed positions (with and without adding the distortion to limb measurement) to SAT317 ephemeris predictions.

satellite in the image, and finally the number of detected stars in the image. All the angle variables are given in degrees. Figure 5 shows a small sample of the Mimas and Enceladus astrometric observations.

We separately provide the pixel coordinates of the satellite centre and the camera pointing information in terms of the right ascension, declination $\left(\alpha_{\mathrm{c}}, \delta_{\mathrm{c}}\right)$ and twist angle $\theta$ in order to give the user the option as to whether to adopt this paper's satellite measurement or pointing method, or their own. They could, for example, use our pointing correction and their own preferred centre-of-figure finding technique, or vice versa.

\subsection{Analysis of results}

Observation residuals relative to JPL ephemerides SAT317 and SAT351 are shown in Figs. 6-9, for Mimas and Enceladus respectively, where the residuals in $\alpha \cos \delta$ and $\delta$ have been converted to kilometres. Table 6 lists the corresponding mean and standard deviation values for these residuals (also in kilometres). In Figs. 10 and 11 we show the differences $\mid \mathrm{O}-\mathrm{C}_{\text {SAT317 }}-$ $|\mathrm{O}-\mathrm{C}|_{\text {SAT351 }}$ for Mimas and Enceladus, respectively. If this difference is positive then SAT317 has a larger residual than SAT351, and vice versa. The mean value of this difference is given in Table 5. From these figures and from Tables 5 and 6 we cannot conclude definitively which of these Mimas ephemerides is better. Nevertheless, we notice that the ephemeris for Enceladus has been updated to a new version, SAT351. The SAT317 ephemeris used Cassini data until August 2009 while the ephemeris SAT351 used data until the end of 2011. Comparing to the observations published in this paper, 39 and 127 astrometric observations of Mimas and Enceladus, respectively, were added since August 2009.

From Table 7, where we show the percentage of residuals lower than their estimated observation uncertainties, we note that these have been better-estimated for Enceladus than for Mimas (we would expect about $66.7 \%$ of the observations to have $(\mathrm{O}-\mathrm{C})<1 \sigma)$. Two possibilities could explain this: since the surface of Mimas is more heavily-cratered than Enceladus, the limb-fitting is likely to be less accurate for Mimas and thus the corresponding uncertainty in the limb-fitting for Mimas could be an underestimate. On the other hand, the precision of the ephemeris for Enceladus is higher than for Mimas. In fact, the results from Table 7 agree with those in Table 6, with both tables confirming that the ephemeris precision is lower for Mimas than for Enceladus.

Cassini imaging has provided some particularly wellresolved images of the Saturnian satellites, allowing very 
Table 4. Sample of the results given by the astrometric reduction.

\begin{tabular}{|c|c|c|c|c|c|c|c|c|c|c|c|c|c|}
\hline Image name & Date & $\begin{array}{l}\text { Time } \\
\text { (UTC) }\end{array}$ & Body & $\begin{array}{c}\alpha \\
\text { (degrees) }\end{array}$ & $\begin{array}{c}\delta \\
\text { (degrees) }\end{array}$ & $\begin{array}{c}\sigma_{\alpha} \times \cos (\delta) \\
\text { (degrees) }\end{array}$ & $\begin{array}{c}\sigma_{\delta} \\
\text { (degrees) }\end{array}$ & $\begin{array}{c}\alpha_{\mathrm{c}} \\
\text { (degrees) }\end{array}$ & $\begin{array}{c}\delta_{\mathrm{c}} \\
\text { (degrees) }\end{array}$ & $\begin{array}{c}\text { Twist angle } \\
\text { (degrees) }\end{array}$ & $\begin{array}{l}\text { Sample } \\
\text { (pixels) }\end{array}$ & $\begin{array}{c}\text { Line } \\
\text { (pixels) }\end{array}$ & $*$ \\
\hline N1479200258 & 04 Nov. 15 & $32: 05.945$ & IMAS & .979909 & 0.0000 & $687 \times 10^{-4}$ & $62 \times 10^{-4}$ & .70 & 761319 & 91.115937 & 281.28 & 729.98 & 11 \\
\hline N1481705814 & 04 Dec. 14 & :31:05.670 & IMAS & 7.870401 & -3.690718 & $9 \times 10^{-4}$ & $359 \times$ & 107.870317 & -3.733118 & 353.939497 & 498.21 & 634.34 & 29 \\
\hline N1484517118 & 2005 Jan. 15 & $21: 25: 51.308$ & MIMAS & 175.477709 & 0.093303 & $0.129 \times 10^{-3}$ & $0.207 \times 10^{-3}$ & 175.489457 & 0.092305 & 15.269756 & 545.29 & 505.29 & 3 \\
\hline N1521712680 & 2006 Mar. 22 & $09: 27: 52.503$ & MIMAS & 288.745684 & 1.988585 & $0.274 \times 10^{-4}$ & $0.283 \times 10^{-4}$ & 288.783017 & 2.008344 & 5.899863 & 613.73 & 443.05 & 51 \\
\hline N1575313583 & 2007 Dec. 02 & $18: 30: 33.311$ & IMAS & 43.610960 & -4.158628 & $0.901 \times 10^{-4}$ & $0.886 \times 10^{-4}$ & 243.619856 & -4.155943 & 282.100264 & 524.57 & 535.14 & 6 \\
\hline N1595040885 & 2008 Jul. 18 & $02: 16: 36.158$ & MIMAS & 141.777941 & -11.025407 & $0.686 \times 10^{-4}$ & $0.687 \times 10^{-4}$ & 141.781475 & -11.021131 & 45.630431 & 509.66 & 495.56 & 8 \\
\hline N1634636975 & 2009 Oct. 19 & 09:06:44.682 & MIMAS & 272.365301 & 3.554427 & $0.416 \times 10^{-4}$ & $0.392 \times 10^{-4}$ & 272.359718 & 3.536634 & 273. & 458.67 & 498.81 & 24 \\
\hline N1648319659 & 2010 Mar. 26 & $17: 49: 50.716$ & MIMAS & 275.565618 & 3.248451 & $0.515 \times 10^{-4}$ & $0.496 \times 10^{-4}$ & 275.5 & 3.292250 & 27 & 643.08 & 547.78 & 15 \\
\hline N1675518282 & 2011 Feb. 04 & $12: 57: 06.091$ & MIMAS & 261.632816 & 4.465046 & 0.80 & $0.875 \times 10^{-4}$ & 261.636276 & 4.462652 & 243.369096 & 500.76 & 517.36 & 6 \\
\hline N1466514680 & 2004 Jun. 21 & $12: 47: 10.340$ & NCELADUS & 38.588063 & 10.206353 & $0.117 \times 10^{-3}$ & $0.111 \times 10^{-3}$ & 38.574930 & 10.205660 & 0.137024 & 473.84 & 513.61 & 3 \\
\hline N1484577892 & 2005 Jan. 16 & $14: 18: 44.852$ & ENCELADUS & 318.438532 & 1.777562 & $0.689 \times 10^{-4}$ & $0.670 \times 10^{-4}$ & 318.433691 & 1.730987 & 6.287934 & 512.35 & 647.95 & 10 \\
\hline N1525360749 & 2006 May 03 & $14: 48: 39.541$ & ENCELADUS & 318.048782 & -1.138369 & $0.790 \times 10^{-4}$ & $0.785 \times 10^{-4}$ & 318.060314 & -1.111871 & 342.129492 & 567.17 & 448.32 & 6 \\
\hline N1572040488 & 2007 Oct. 25 & $21: 19: 21.288$ & ENCELADUS & 133.153457 & 1.006058 & $0.114 \times 10^{-3}$ & $0.961 \times 10^{-4}$ & 133.162424 & 1.033192 & 353.446634 & 546.48 & 435.93 & 4 \\
\hline N1584025375 & 2008 Mar. 12 & $14: 26: 03.829$ & ENCELADUS & 345.192303 & -71.883886 & $0.736 \times 10^{-4}$ & $0.742 \times 10^{-4}$ & 345.246903 & -71.883564 & 81.855844 & 517.56 & 462.39 & 8 \\
\hline N1636982497 & 2009 Nov. 15 & $12: 38: 29.837$ & ENCELADUS & 293.373282 & 1.521043 & $0.608 \times 10^{-4}$ & $0.621 \times 10^{-4}$ & 293.372193 & 1.515141 & 276.126094 & 494.06 & 510.18 & 10 \\
\hline & 2010 Sep. 23 & $14: 31: 14.194$ & ENCELADUS & 193.921811 & 3.487161 & & $0.738 \times 10^{-4}$ & 193.923545 & 3.490967 & 263.050203 & 521.90 & 517.85 & 7 \\
\hline N1675108551 & 2011 Jan. 30 & 19:08:16.839 & ENCELADUS & 342.949825 & -3.660910 & $0.935 \times 10^{-4}$ & $0.727 \times 10^{-4}$ & 342.947282 & -3.759135 & 185.366996 & 492.09 & 225.83 & 8 \\
\hline
\end{tabular}

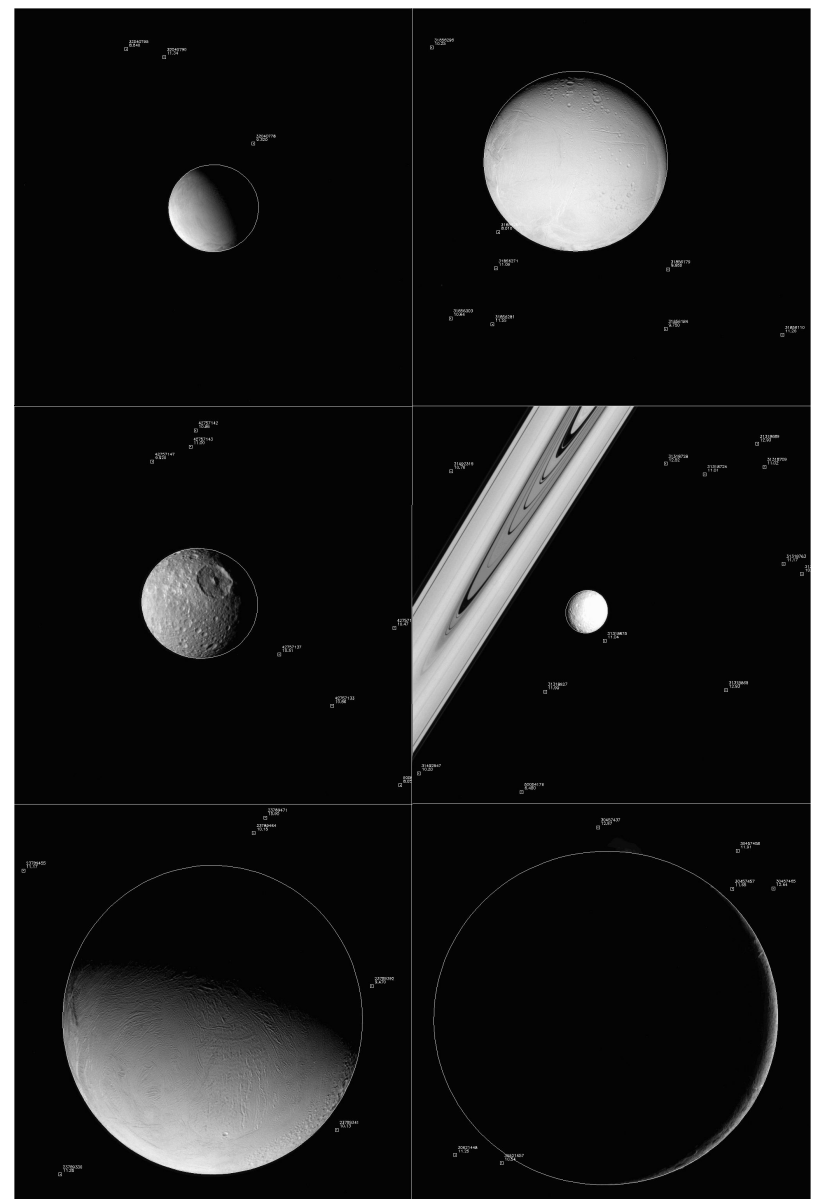

Fig. 5. Examples of Mimas (middle) and Enceladus (top and bottom) images used in this study. Images names: N1484519176, N1487264883, N1501627117, N1514074586, N1569849851 and N1669795989, respectively. Expected positions of UCAC2 stars are superimposed on the images.

accurate astrometric positions to be obtained. A high number of stars combined with high satellite resolution inevitably leads to better astrometric accuracy, however such an optimal combination is seldom achieved. Figures 12 and 13 show the variation of the number of stars versus the observed satellite's size (in pixels) in a $1024 \times 1024$ pixel image for all the observations of Mimas and Enceladus, respectively. From these figures we see how the

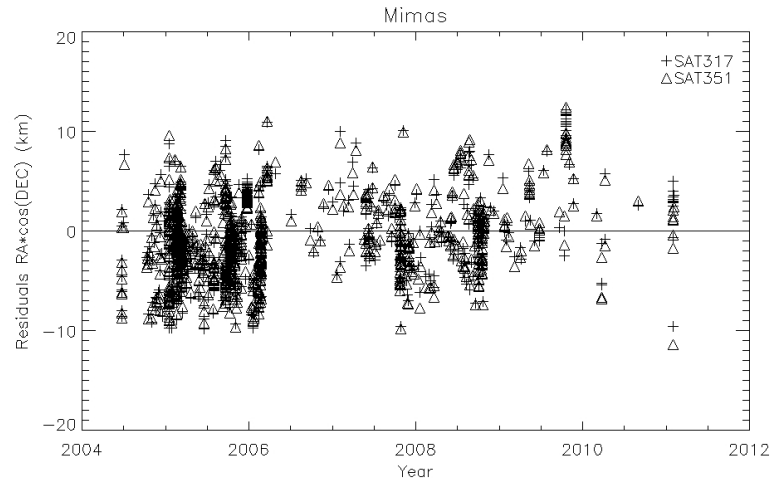

Fig. 6. Residuals for observed positions of Mimas relative to JPL predictions in $\alpha \cos \delta$ converted to kilometres. Crosses show residuals relative to SAT317 and triangles residuals relative to SAT351.

Table 5. Mean difference $\left\langle|\mathrm{O}-\mathrm{C}|_{\mathrm{SAT} 317}-|\mathrm{O}-\mathrm{C}|_{\mathrm{SAT} 351}\right\rangle$ in $\alpha \cos \delta$ and $\delta$ for Mimas and Enceladus.

\begin{tabular}{lcc}
\hline \hline & \multicolumn{2}{c}{$\left\langle|\mathrm{O}-\mathrm{C}|_{\text {SAT317 }}-\mid \mathrm{O}-\mathrm{C}_{\text {SAT351 }}\right\rangle$} \\
& $\alpha \cos \delta(\mathrm{km})$ & $\delta(\mathrm{km})$ \\
\hline Mimas & 0.09 & -0.39 \\
Enceladus & 0.17 & 0.26 \\
\hline
\end{tabular}

number of stars decreases as the satellite's resolution increases. This is expected since the stars tend to be hidden behind the image of the satellite in the higher resolution images, so that their detection probability is correspondingly lower. Thus, the observational uncertainty decreases with the increase in the number of detected stars in the image, as shown in Figs. 14 and 15 for Mimas and Enceladus, respectively. We also notice other effects in these figures and note that the error in the detections of the stars is only one of the three main sources of error, along with the error in the spacecraft's position error and the satellite's centreof-figure measurement error. We discuss these sources of error in more detail later in this section.

Since the ellipse fitting was performed using only the visible limb of the satellite, we studied the behaviour of the residuals as a function of phase angle. Figure 16 shows position residuals in kilometres for Mimas and Enceladus compared to the SAT351 ephemeris, in a frame in which the $X$-axis is oriented in the sun direction. Table 8 shows the corresponding mean residuals and standard deviations for both satellites in this 


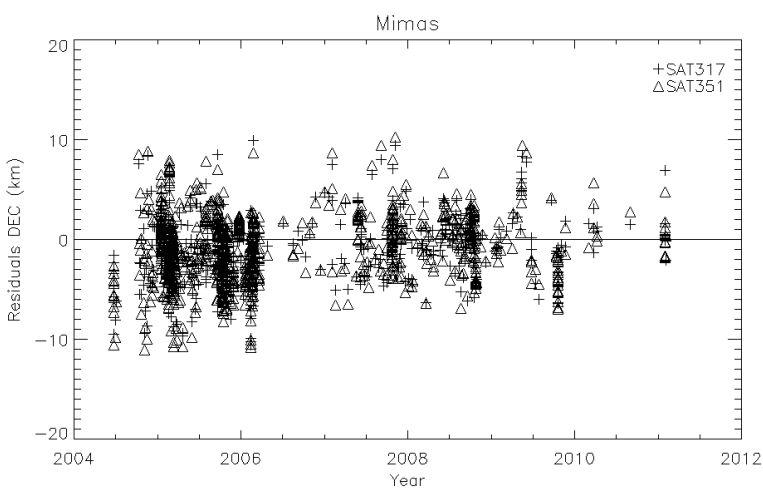

Fig. 7. Residuals for observed positions of Mimas relative to JPL predictions in $\delta$ converted to kilometres. Crosses show residuals relative to SAT317 and triangles residuals relative to SAT351.

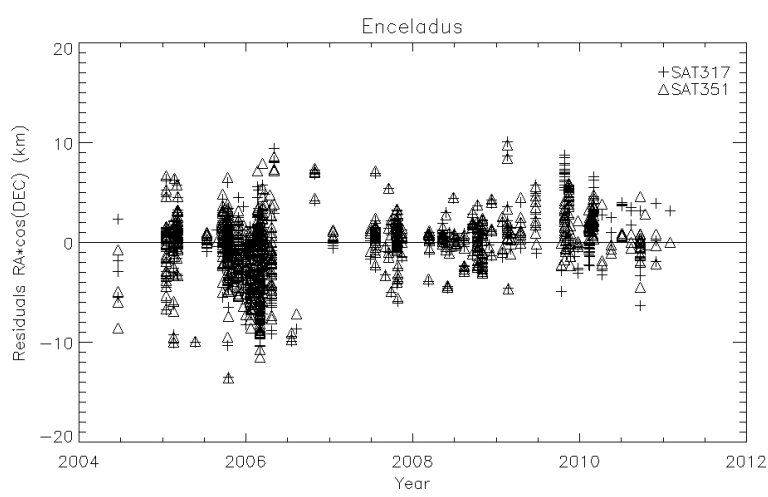

Fig. 8. Residuals for observed positions of Enceladus relative to JPL predictions in $\alpha \cos \delta$ converted to kilometres. Crosses show residuals relative to SAT317 and triangles residuals relative to SAT351.

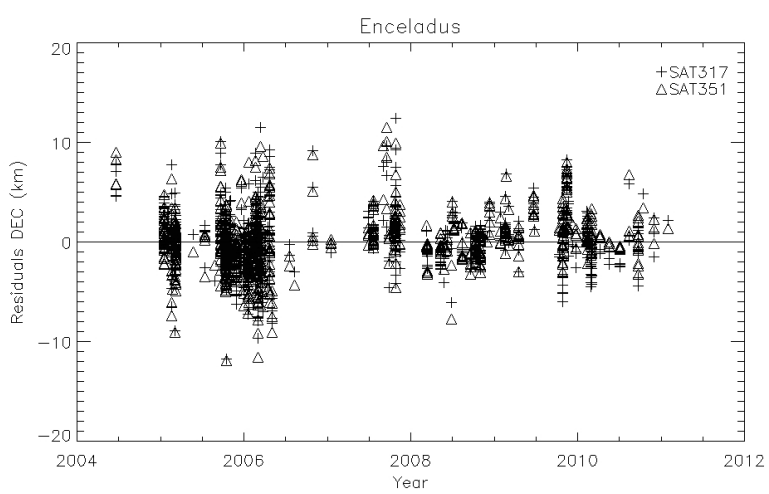

Fig. 9. Residuals for observed positions of Enceladus relative to JPL predictions in $\delta$ converted to kilometres. Crosses show residuals relative to SAT317 and triangles residuals relative to SAT351.

frame. It is clear from Fig. 16 and especially from Table 8 that the observed positions for both satellites are shifted towards the sun, compared to their computed positions. However, the shifts and the standard deviations are more significant for Mimas than for Enceladus. Although Mimas' ephemeris is less precise than Enceladus', the surface of Mimas is also more heavily cratered than Enceladus' and therefore the phase effect could be more important for Mimas. Another possible explanation is that the dimensions of Mimas could be larger than those based on the shape model given in Thomas et al. (2007). This could also lead to such a shift.

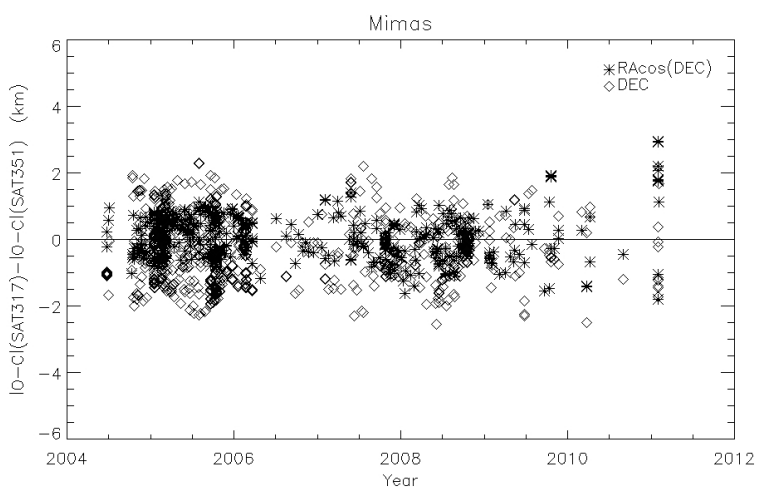

Fig. 10. Difference between residuals for Mimas compared to SAT317 and those compared to SAT351 $|\mathrm{O}-\mathrm{C}|_{\mathrm{SAT} 317}-|\mathrm{O}-\mathrm{C}|_{\mathrm{SAT} 351}$, with diamonds showing residual differences for $\alpha \cos \delta$ and stars for $\delta$.

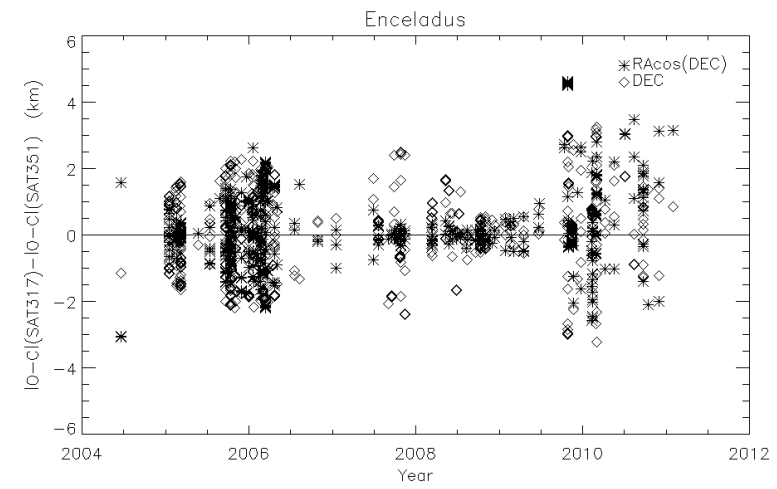

Fig. 11. Difference between residuals for Enceladus compared to SAT317 and those compared to SAT351 $|\mathrm{O}-\mathrm{C}|_{\text {SAT317 }}-\mid \mathrm{O}-\mathrm{C}_{\text {SAT351 }}$, with diamonds showing residual differences for $\alpha \cos \delta$ and stars for $\delta$.

Table 6. Mean values of residuals with respect to the JPL SAT317 and SAT351 ephemerides in kilometres, including standard deviations.

\begin{tabular}{lcccc}
\hline \hline & $\langle\alpha \cos \delta\rangle(\mathrm{km})$ & $\sigma_{\alpha \cos \delta}(\mathrm{km})$ & $\langle\delta\rangle(\mathrm{km})$ & $\sigma_{\delta}(\mathrm{km})$ \\
\hline Mimas (SAT317) & -0.83 & 4.15 & -1.02 & 2.95 \\
Mimas (SAT351) & -0.86 & 4.01 & -0.91 & 3.39 \\
Enceladus (SAT317) & -0.35 & 3.20 & 0.17 & 2.99 \\
Enceladus (SAT351) & -0.25 & 3.00 & 0.04 & 2.78 \\
\hline
\end{tabular}

Table 7. Percentage of observations with $(\mathrm{O}-\mathrm{C})$ less than the observation uncertainty $\sigma$ in $\alpha \cos \delta$ and in $\delta$.

\begin{tabular}{lcc}
\hline \hline & $\%(\mathrm{O}-\mathrm{C})_{\alpha \cos \delta}<\sigma_{\alpha \cos \delta}$ & $\%(\mathrm{O}-\mathrm{C})_{\delta}<\sigma_{\delta}$ \\
\hline Mimas (SAT317) & 47.2 & 63.6 \\
Mimas (SAT351) & 48.5 & 53.8 \\
Enceladus (SAT317) & 60.4 & 57.9 \\
Enceladus (SAT351) & 61.0 & 66.6 \\
\hline
\end{tabular}

In Fig. 17, we show the twist angle correction (in degrees) as a function of the number of stars used in its computation. It is clear from this figure that the twist angle correction converges to a precise value as the number of stars increases. This is to be expected since a higher number of stars is needed to obtain a more precise value of the correction. We used a total of $294 \mathrm{im}-$ ages from the same star cluster image series (from N1580739191 to N1637129711) to calibrate this parameter while setting the scale factor to $\rho=1.2354 \mathrm{arcsec} / \mathrm{pixel}$. These images have been taken over a period of time of about 1.8 years. We obtained a mean twist angle correction value of about $-9.6 \times 10^{-2}$ degrees 


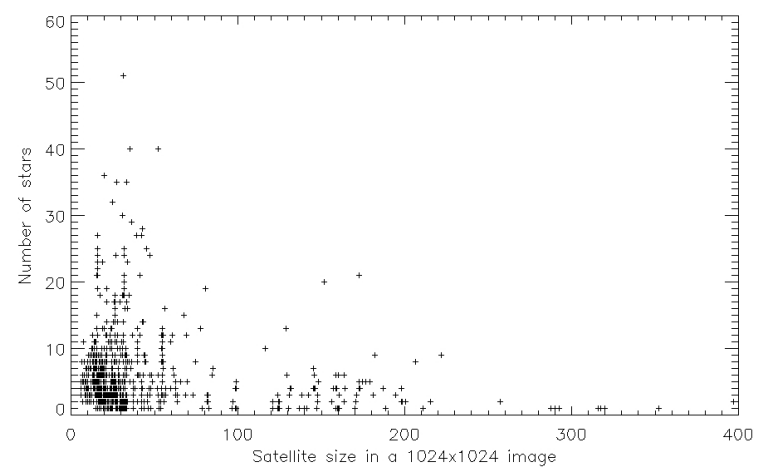

Fig. 12. Plot showing how the possibility of star detections decreases with increase of Mimas size in the image. In $X$ axis is the satellite size and in the $Y$ axis is the number of stars.

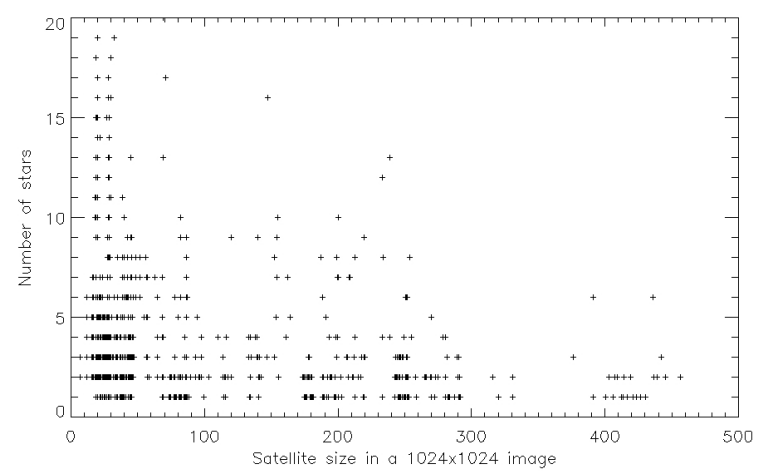

Fig. 13. Plot showing how the possibility of star detections decreases with increase of Enceladus size in the image. In $X$ axis is the satellite size and in the $Y$ axis is the number of stars.

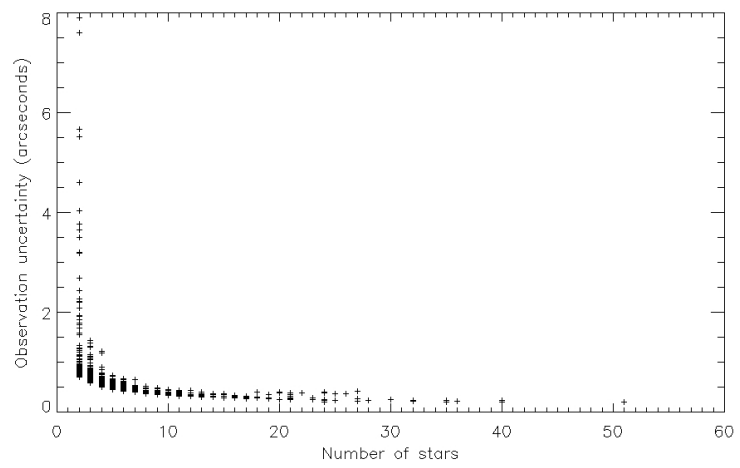

Fig. 14. Evolution of the observation uncertainty $(\sigma=$ $\left.\sqrt{\left(\sigma_{\alpha} \times \cos \delta\right)^{2}+\left(\sigma_{\delta}\right)^{2}}\right)$ on the position of Mimas versus the number of detected stars per image.

with a standard deviation of $5.1 \times 10^{-3}$ degrees. Here we have used the spacecraft frame defined in the JPL SPICE-system as "Cassini_SC_COORD", while Cassini's NAC frame is named "Cassini_ISS_NAC". For comparison, the angular difference between the "north" axes of these two frames, computed directly using the SPICE system, is $-9.1 \times 10^{-2}$ degrees, while our value has been obtained using star measurements (independently of the SPICE library). If using the "Cassini_SC_COORD" frame, this constant correction must be added to convert the camera's twist angle to the NAC-centred frame. A useful added benefit of our empirical measurement of this quantity is that it has allowed

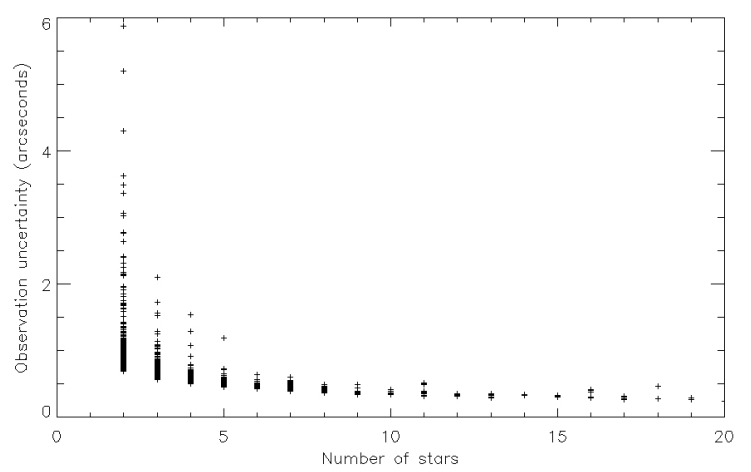

Fig. 15. Evolution of the observation uncertainty $(\sigma=$ $\sqrt{\left.\left(\sigma_{\alpha} \times \cos \delta\right)^{2}+\left(\sigma_{\delta}\right)^{2}\right)}$ on the position of Enceladus versus the number of detected stars per image.

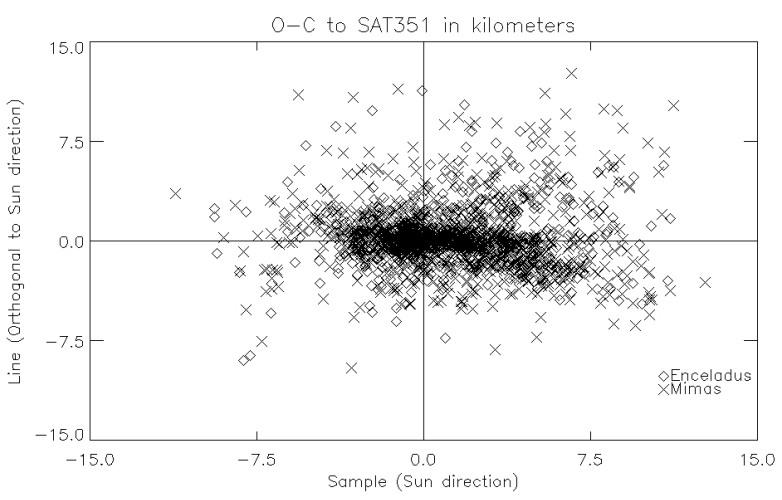

Fig. 16. Residuals of Mimas and Enceladus to SAT351 in kilometres represented in a frame where $X$ is in the sun direction. " $X$ " denotes for Mimas and "diamonds" for Enceladus.

Table 8. Mean residuals to SAT351 in kilometres represented in a frame where $X$ is in the sun direction.

\begin{tabular}{lcccc}
\hline \hline & $\langle\mathrm{O}-\mathrm{C}\rangle_{X}(\mathrm{~km})$ & $\langle\mathrm{O}-\mathrm{C}\rangle_{Y}(\mathrm{~km})$ & $\sigma_{(\mathrm{O}-\mathrm{C})_{X}}(\mathrm{~km})$ & $\sigma_{(\mathrm{O}-\mathrm{C})_{Y}}(\mathrm{~km})$ \\
\hline Mimas & 1.52 & 0.17 & 3.80 & 2.77 \\
Enceladus & 0.72 & 0.25 & 2.99 & 2.01 \\
Both & 1.11 & 0.21 & 3.43 & 2.41 \\
\hline
\end{tabular}

Notes. The first line gives the residuals for Mimas, the second for Enceladus and the last for both satellites.

us to determine the error in the camera's twist angle: we obtain about $5.1 \times 10^{-3}$ degrees.

Finally, in Figs. 18 and 19 we show the residuals in sample and line, respectively, for Mimas and Enceladus versus each satellite's location in a given image. We see from these plots that for the majority of images, the satellite was located around the centre of the field-of-view, which is to be expected, since in most cases the satellites in question were specifically targeted. However, the lack of systematic trends in these plots provides assurance that the camera has been sufficiently well-modelled.

\subsection{Sources of error}

Each observation has three main sources of errors: 1) the pointing correction $\sigma_{\alpha_{\mathrm{p}}}$ and $\sigma_{\delta_{\mathrm{p}}}, 2$ ) finding the centre-of-figure of the satellite $\sigma_{\mathrm{c}}$ and 3) the spacecraft position $\sigma_{\mathrm{sp}}$.

1) As described previously, the pointing correction is derived by comparing the imaged positions of background stars with 


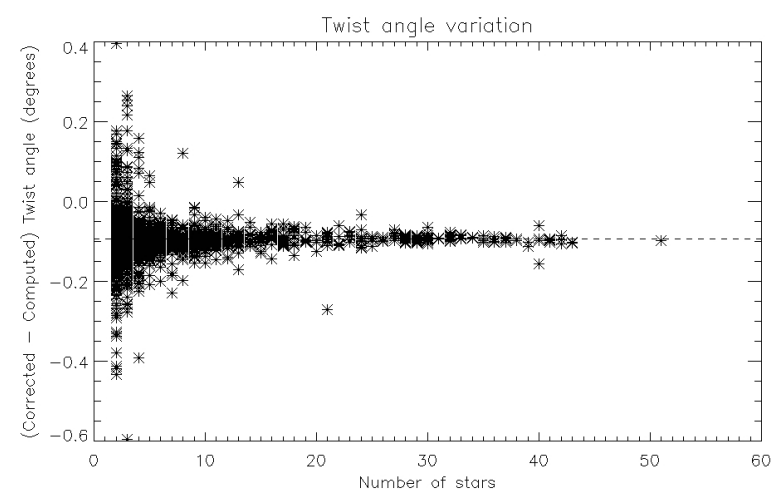

Fig. 17. Difference between the corrected value of the twist angle by stars and the computed one by SPICE library, versus the number of stars. The dashed line is the mean value.

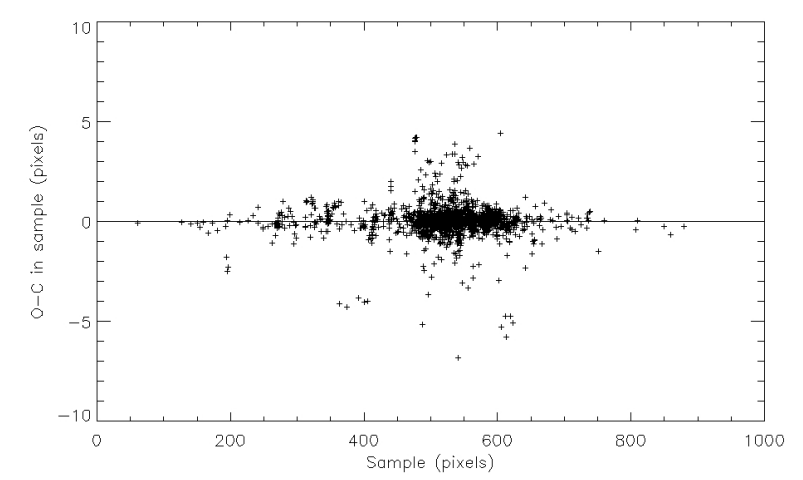

Fig. 18. Residuals of Mimas and Enceladus positions in sample (pixels) versus their sample location in the image.

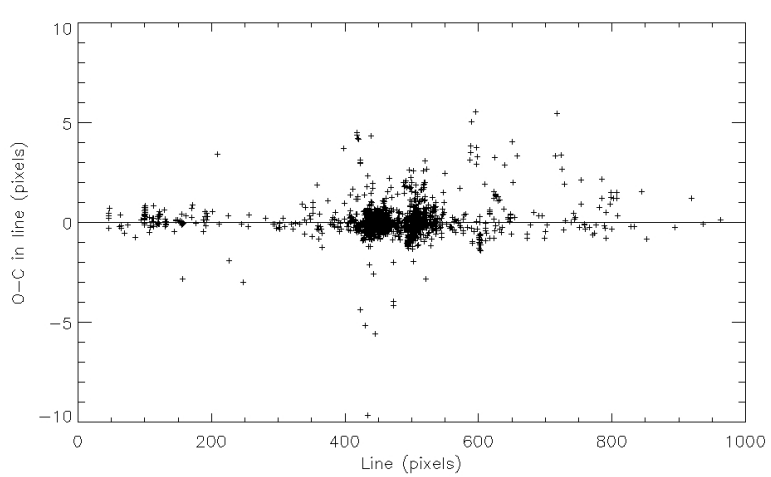

Fig. 19. Residuals of Mimas and Enceladus positions in line (pixels) versus their line location in the image.

their equivalent positions obtained from a reference star catalogue (the UCAC2 catalogue in this work). Potential sources of error for the pointing correction are therefore the technique used to detect the stars in the image, and inherent errors in the star catalogue itself. Star detection was performed using the "FIND" method (Stetson 1987), which searches for Gaussian signals in the image with user-defined full width at half maximum (FWHM, for the Cassini NAC, we used $F W H M=1.3$ pixels). It is noteworthy that the "FIND" routine does not do a full weighted least-squares fit. Instead, it provides approximate image centres, which are themselves intended as starting estimates for a future full non-linear least squares profile fit. On their own, the centroids provided by FIND are probably precise to $1 / 3$ to $1 / 4$ of a pixel. To

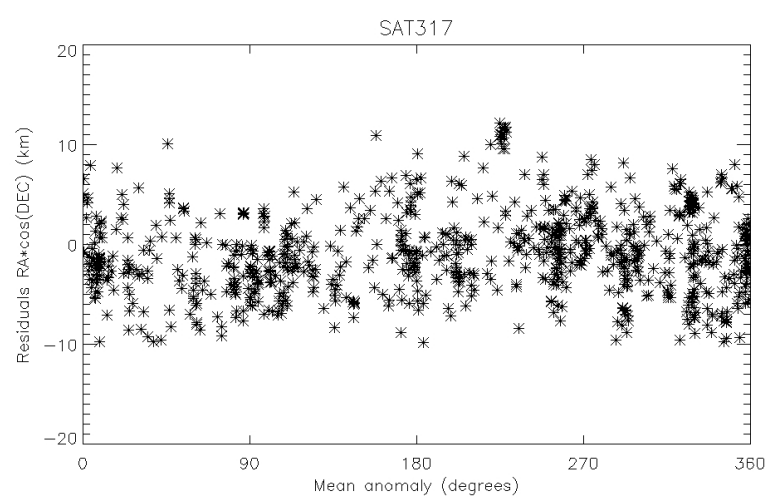

Fig. 20. Residuals in Mimas' observed positions relative to the JPL SAT317 ephemeris in $\alpha \cos \delta$ converted to kilometres and plotted versus Mimas' mean anomaly.

achieve a precision of order FWHM/(S/N), a proper pointspread function would need to be provided for each image, followed by a full least-squares solution (Peter Stetson, private comm). Hence, the star detection method could be modified in this way in the future, in order to measure star positions with better precision. Also, since this routine does not provide an uncertainty for each measured star position and until a full least-squares method is adopted, we decided to set the star detection uncertainty to $\sigma_{\text {extraction }}=F W H M / 2.355$. Errors in the reference star coordinates $\left(\sigma_{\alpha_{*}}, \sigma_{\delta_{*}}\right)$ are provided in the UCAC2 catalogue. Finally, the error in $\alpha$ and $\delta$ due to the pointing solution is a function not only of the number of stars in the field but also of their location and of the location of the satellite. The effect of an error in the camera's twist angle is generally lower near the image centre and increases towards the corners of the image.

2) Each point on the detected limb has a measurement error of 0.5 pixels, while the errors in a given satellite's 3-D shape are provided in Thomas et al. (2007). Hence, these latter form the principle sources of error for the determination of the centre-of-figure of the satellite, $\sigma_{X \text { cen }}$ and $\sigma_{Y \text { cen }}$.

3) The uncertainty in the spacecraft's position is estimated to about 100 metres, which causes an additional source of error on the observed position of the satellite.

The method used to estimate observational uncertainty is described in Appendix A.

\subsection{Frequency analysis}

The residuals between the observed positions and those derived from JPL ephemerides SAT317 and SAT351 for both Mimas and Enceladus were subject to further analysis. A frequency analysis of the Mimas residuals in $\alpha \cos \delta$ given in Fig. 6 shows a periodic signal of $\approx 1.8 \mathrm{~km}$ with a period of 0.9443 days. In Figs. 20 , 21 , we also show the residuals for Mimas (in $\alpha \cos \delta$ ) relative to SAT317 and SAT351 respectively, versus Mimas' mean anomaly. From this figure we see a periodic bias in $\alpha \cos \delta \operatorname{com}$ pared to the respective ephemerides. However, we have not detected any periodic variations in the residuals shown in Figs. 7-9.

To investigate a possible origin for the detected periodic signal described above, we also performed a frequency analyse of the variation with time of Mimas' mean longitude derived from the JPL ephemeris, SAT351. Based on this analysis, we detected a short-term variation, with a period of 0.9449 days and amplitude of $33 \mathrm{~km}$. Similar values were obtained using the NOE-6-12 ephemeris (Lainey et al. 2012) and the TASS1.7 orbital model 


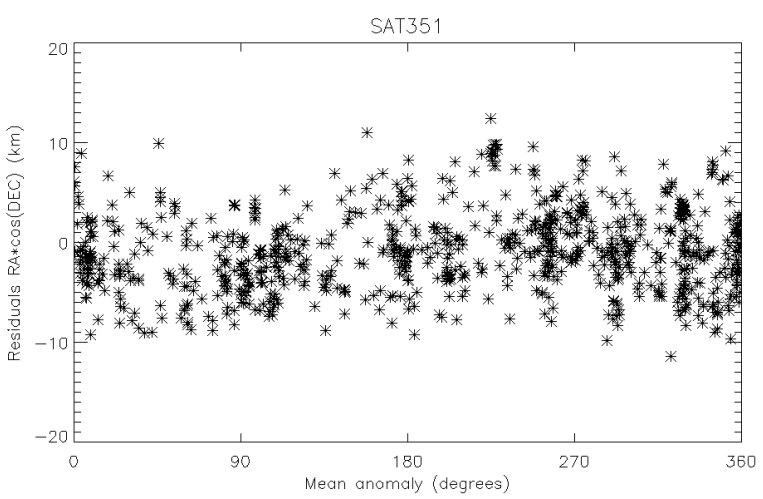

Fig. 21. Residuals in Mimas' observed positions relative to the JPL SAT351 ephemeris in $\alpha \cos \delta$ converted to kilometres and plotted versus Mimas' mean anomaly.

(Duriez \& Vienne 1997 and Vienne \& Duriez 1995). However, the difference between the measured period in the residuals and the period detected in these models is about $6 \times 10^{-4}$ days, which could be within the uncertainties of the frequency analysis. This periodic bias in the Mimas observations must be attributable to errors in the initial conditions of the integration (including the masses of Saturn and of the satellites) or to unmodeled perturbations, perhaps from very small satellites, the rings, or higherorder harmonics in Saturn's gravity field.

In both the SAT351 and NOE-6-12 ephemerides we also found two additional periods in mean longitude of 1.0016 days and 0.9424 days, with amplitudes of $255 \mathrm{~km}$ and $13 \mathrm{~km}$, respectively, that do not appear in the TASS model (see Table 9). The period of 0.9424 days is the orbital period of Mimas, while the period of the resulting "beat frequency" between the 0.9424 and 1.0016 days signals is 15.94 days, which is the orbital period of Titan. suggesting that some terms could be missing in this model, including Titan's effect on Mimas' orbit. This is consistent with Peng et al. (2008) who found a significant periodic signal in their Mimas $(\mathrm{O}-\mathrm{C})$ residuals compared to the TASS1.7 ephemeris, based on ground-based astrometric observations.

\section{Conclusion}

In this paper, we provided 1790 astrometric observations of two of the main saturnian satellites, Mimas and Enceladus, using Cassini ISS images. We developed an alternative model to that of Owen (2003) and shown that our model can also provide accurate satellite astrometry while at the same time (unlike then Owen model) being easily invertible. We used images of star clusters to calibrate the scale factor in our model that converts from arcseconds to pixels, obtaining a value of $\rho=1.2354$ arcsec/pixel. We conclude that a square pixel shape is a reasonable approximation for the purposes of satellite astrometry using the Cassini ISS NAC. Finally, to determine the satellite's centr-offigure, methods for limb measurement and ellipse fitting were developed. We showed also that distortion does not significantly affect the satellite's measured position.

Using the methods described in this paper, we were able to reduce 870 out of about 2500 available NAC images of Mimas and 920 out of about 5500 of available NAC images of Enceladus. Comparing the observed-minus-computed residuals relative to the JPL ephemerides SAT317 and SAT351, we conclude that neither ephemeris provides significantly better results than the other for Mimas, while for Enceladus, the residuals for SAT351 are smaller than those relative to SAT317. We also
Table 9. Detected short periods from an analysis of the mean longitude of Mimas from the TASS, SAT351, and NOE-6-12 orbital models.

\begin{tabular}{lcc}
\hline \hline & Period (days) & Amplitude $(\mathrm{km})$ \\
\hline TASS & 0.9449 & 32 \\
\hline \multirow{3}{*}{ SAT351 } & 1.0016 & 255 \\
& 0.9449 & 33 \\
& 0.9424 & 13 \\
\hline \multirow{3}{*}{ NOE-6-12 } & 1.0016 & 255 \\
& 0.9449 & 33 \\
& 0.9424 & 13 \\
\hline
\end{tabular}

found that the precision of Enceladus for both ephemerides is better than that of Mimas.

An analysis of our computed twist-angle corrections showed that this correction can be set to a fixed value, so that solving for this parameter image-by-image can be avoided in future Cassini astrometric reduction.

A frequency analysis of the observed-minus-computed residuals showed a periodic variation for Mimas' residuals in $\alpha \cos \delta$ of 0.9443 days with an amplitude of $\approx 1.8 \mathrm{~km}$. A further frequency analysis of the mean longitude derived from the TASS, SAT351 and NOE ephemerides leads us to conclude that this period may correspond to one of the known short period terms of 0.9449 days in these orbital models. We also found that two particular short-period effects were absent in the TASS analysis when compared to SAT351 and NOE-6-12, suggesting that the terms corresponding to these frequencies may be missing in the TASS orbital model.

Finally, we conclude that, with an observational accuracy of a few kilometres, Cassini's NAC high resolution imaging has proven its worth and importance for astrometric reduction and orbital modelling.

Acknowledgements. The authors woud like to thank Vincent Robert and Alain Vienne for their assistance. The authors also thank the anonymous reviewer for very useful and helpful comments. This work has been funded by UPMCEMERGENCE (contract number EME0911). This work was also supported by the Science and Technology Facilities Council (grant number ST/F007566/1) and by an International Exchange grant from the Royal Society and the CNRS.

\section{Appendix A: Observation uncertainty estimation method}

The observational uncertainties $\sigma_{\alpha} \cos \delta$ and $\sigma_{\delta}$ shown in Table 4 were computed using the following expressions:

$\left(\sigma_{\alpha} \cos \delta\right)^{2}=\left(\sigma_{\alpha_{\mathrm{p}}} \cos \delta_{\mathrm{p}}\right)^{2}+\sigma_{\alpha \mathrm{c}}^{2}+\sigma_{\mathrm{sp}}^{2}$

$\sigma_{\delta}^{2}=\sigma_{\delta_{\mathrm{p}}}^{2}+\sigma_{\delta \mathrm{c}}^{2}+\sigma_{\mathrm{sp}}^{2}$

where,

$$
\begin{aligned}
\left(\sigma_{\alpha_{\mathrm{p}}} \cos \delta_{\mathrm{p}}\right)^{2}= & \left(\frac{\alpha_{s} \cos \delta_{s}}{\rho} \times \sigma_{\rho}\right)^{2}+\left(\delta_{s} \times \sigma_{\theta}\right)^{2} \\
& +\left(\rho \cos (\theta) \times \sigma_{\Delta X}\right)^{2}+\left(\rho \sin \theta \times \sigma_{\Delta Y}\right)^{2} \\
\left(\sigma_{\delta_{\mathrm{p}}}\right)^{2}= & \left(\frac{\delta_{s}}{\rho} \times \sigma_{\rho}\right)^{2}+\left(\alpha \cos \delta_{s} \times \sigma_{\theta}\right)^{2} \\
& +\left(\rho \sin (\theta) \times \sigma_{\Delta X}\right)^{2}+\left(\rho \cos \theta \times \sigma_{\Delta Y}\right)^{2}
\end{aligned}
$$

where $\alpha_{\mathrm{p}}$ and $\delta_{\mathrm{p}}$ are the observed satellite's coordinates, $\sigma_{\rho}$ is given in Table 1 , and finally $\sigma_{\theta}, \sigma_{\Delta X}$ and $\sigma_{\Delta Y}$ are the errors in 
the pointing correction related to rotation and translation, respectively. These latter contain the errors in star extraction $\sigma_{\text {extraction }}$ and those provided in the UCAC2 catalogue $\left(\sigma_{\alpha_{*}}, \sigma_{\delta_{*}}\right)$.

The satellite centre measurement uncertainty $\sigma_{\alpha \mathrm{c}}$ and associated $\sigma_{\delta \mathrm{c}}$ is expressed as follows:

$\sigma_{\alpha \mathrm{c}}^{2}=\left(\rho \cos \theta \times \sigma_{X \mathrm{cen}}\right)^{2}+\left(\rho \sin \theta \times \sigma_{Y \mathrm{cen}}\right)^{2}$

$\sigma_{\delta \mathrm{c}}^{2}=\left(\rho \sin \theta \times \sigma_{X \mathrm{cen}}\right)^{2}+\left(\rho \cos \theta \times \sigma_{Y \mathrm{cen}}\right)^{2}$

$\sigma_{X \text { cen }}$ and $\sigma_{Y \text { cen }}$ are the errors in sample and line from the satellite centre determination based on limb fitting.

Finally, the error in the spacecraft position is,

$\sigma_{\mathrm{sp}}=\arcsin \left(\frac{10^{-1}}{D}\right)$

where $D$ is the distance (in kilometres) between the spacecraft and the observed satellite.

These expressions were derived by differentiating Eq. (4).

\section{Appendix B: Kernels}

The following is the list of SPICE kernels used for astrometric reduction in this paper:

cas_v40.tf

cas_rocks_v18.tf

cpck_rock_11May2009_merged.tpc

pck 00010 .tpc

cpck140ct2011.tpc

naif 0010 .tls

cas 00148 .tsC

091005AP_SCPSE_09248_17265.bsp

110427BP_SCPSE_11117_11133.bsp

110504AP_SCPSE_11124_11133.bsp

110511AP_SCPSE_11127_11178.bsp

110523AP_SCPSE_11143_11178.bsp

100209AP_RE_90165_18018.bsp

Q90202BP_IRRE_Q0256_50017.bsp

Q41014R_SCPSE_Q1066_04199.bsp

041219R_SCPSE_04199_04247.bsp

Q50105RB_SCPSE_04247_04336.bsp

Q50214R_SCPSE_Q4336_Q5015.bsp

Q50411R_SCPSE_05015_05034.bsp

050414RB_SCPSE_05034_05060.bsp

050504R_SCPSE_05060_05081.bsp

Q50506R_SCPSE_05081_05097.bsp

050513RB_SCPSE_05097_05114.bsp

050606R_SCPSE_Q5114_05132.bsp

Q50623R_SCPSE_05132_05150.bsp

050708R_SCPSE_05150_05169.bsp

Q50802R_SCPSE_05169_05186.bsp

Q50825R_SCPSE_05186_05205.bsp

Q50907R_SCPSE_05205_05225.bsp

Q50922R_SCPSE_05225_05245.bsp

Q51011R_SCPSE_05245_05257.bsp Q51021R_SCPSE_05257_05275.bsp Q51114R_SCPSE_05275_05293.bsp Q51213R_SCPSE_05293_05320.bsp Q60111R_SCPSE_05320_05348.bsp Q60213R_SCPSE_05348_06005.bsp 060321R_SCPSE_06005_06036.bsp 060417R_SCPSE_06036_06068.bsp 060515R_SCPSE_06068_06099.bsp 060614R_SCPSE_06099_06130.bsp 060719R_SCPSE_06130_06162.bsp Q60810R_SCPSE_06162_06193.bsp 060907R_SCPSE_06193_06217.bsp 060925R_SCPSE_06217_06240.bsp 061013R_SCPSE_06240_06260.bsp
061108R_SCPSE_06260_06276.bsp 061116R_SCPSE_06276_06292.bsp 061129RB_SCPSE_06292_06308.bsp 061213R_SCPSE_06308_06318.bsp 070109R_SCPSE_06318_06332.bsp Q70117R_SCPSE_06332_06342.bsp 070125R_SCPSE_06342_06356.bsp 070208R_SCPSE_06356_07008.bsp 070213R_SCPSE_07008_07023.bsp 070312R_SCPSE_07023_07042.bsp 070405R_SCPSE_07042_07062.bsp 070430R_SCPSE_07062_07077.bsp 070507R_SCPSE_07077_07094.bsp 070517R_SCPSE_07094_07106.bsp 070605R_SCPSE_07106_07125.bsp 070625R_SCPSE_07125_07140.bsp 070705R_SCPSE_07140_07155.bsp 070727R_SCPSE_07155_07170.bsp 070822R_SCPSE_07170_07191.bsp 071017R_SCPSE_07191_07221.bsp 071127R_SCPSE_07221_07262.bsp 080117R_SCPSE_07262_07309.bsp 080123R_SCPSE_07309_07329.bsp 080225R_SCPSE_07329_07345.bsp 080307R_SCPSE_07345_07365.bsp 080327R_SCPSE_07365_08045.bsp 080428R_SCPSE_08045_08067.bsp 080515R_SCPSE_08067_08078.bsp 080605R_SCPSE_08078_08126.bsp 080618R_SCPSE_08126_08141.bsp 080819R_SCPSE_08141_08206.bsp 080916R_SCPSE_08206_08220.bsp 081031R_SCPSE_08220_08272.bsp 081126R_SCPSE_08272_08294.bsp 081217R_SCPSE_08294_08319.bsp Q90120R_SCPSE_08319_08334.bsp 090202R_SCPSE_08334_08350.bsp 090225R_SCPSE_08350_09028.bsp Q90423R_SCPSE_09028_09075.bsp 090507R_SCPSE_09075_09089.bsp Q90520R_SCPSE_09089_09104.bsp 090609R_SCPSE_09104_09120.bsp 090624R_SCPSE_09120_09136.bsp Q90701R_SCPSE_09136_09153.bsp 090708R_SCPSE_09153_09168.bsp 090806R_SCPSE_09168_09184.bsp 090817R_SCPSE_09184_09200.bsp 090921R_SCPSE_09200_09215.bsp 090924R_SCPSE_09215_09231.bsp Q91116R_SCPSE_Q9231_09275.bsp 091208R_SCPSE_09275_09296.bsp 100107R_SCPSE_09296_09317.bsp 100113R_SCPSE_09317_09339.bsp 100114R_SCPSE_09339_09355.bsp 100127R_SCPSE_09355_10003.bsp 100209R_SCPSE_10003_10021.bsp 100325R_SCPSE_10021_10055.bsp 100420R_SCPSE_10055_10085.bsp 100519R_SCPSE_10085_10110.bsp 100616R_SCPSE_10110_10132.bsp 100625R_SCPSE_10132_10146.bsp 100706R_SCPSE_10146_10164.bsp 100726R_SCPSE_10164_10178.bsp 100913R_SCPSE_10178_10216.bsp 101013R_SCPSE_10216_10256.bsp 101210R_SCPSE_10256_10302.bsp 101215R_SCPSE_10302_10326.bsp 110224R_SCPSE_10326_10344.bsp 110204R_SCPSE_10344_11003.bsp 110308R_SCPSE_11003_11041.bsp 110504R_SCPSE_11041_11093.bsp 110519R_SCPSE_11093_11119.bsp 04009_04051pg.bc 04051_04092pg.bc 04092_04135pg.bc 04135_04171pc_psiv2.bc 04171_04212ra_s02.bc 04212_04256ra_s03.bc 
Q4256_Q4292pd_S4_psiv2.bc Q4292_04320pf_fsiv.bc Q4320_04351pe_fsiv.bc Q4276_04281ra.bc

04316_04321ra.bc

Q4351_05022pe_psiv2_merge.bc 05022_05058pg_fsiv1.bc 05058_05099pg_psiv2.bc 05099_05134pe_psiv2.bc 05122_05125pc_fsiv.bc 05134_05169ph_fsiv_reva.bc 05137_05142ra.bc 05169_05212pf_psiv2.bc Q5212 05242pk fsiv.bc 05242_05281pf_psiv2.bc Q5281_05316pe_PSIV2.bc 05316_05351pd_psiv2.bc 05351_06027pe_psiv2.bc 06027_06070pd_psiv2.bc 06070_06112pd_psiv2.bc 06070_06112pe_fsiv.bc 06112_06154pe_psiv2.bc 06154_06198pc_psiv2.bc 06198_06231pf_fsiv.bc 06231_06263pd_psiv2.bc 06263_06295pc_psiv2.bc 06295_06328pf_fsiv.bc 06328_07005pa_psiv2.bc 07005_07048pd_psiv2.bc 07048_07087pd_psiv2.bc 07087_07124pe_psiv2.bc 07124_07162pe_psiv2.bc 07162_07195pe_psiv2.bc 07162_07163ra.bc 07195_07223pd_psiv2.bc 07223_07265pe_psiv2.bc 07265_07304pc_psiv2.bc 07304_07348pe_fsiv.bc 07348_08022pf fsiv.bc 08022_08047pe_psiv2.bc 08047_08083pb_psiv2.bc 08057 08062ra.bc

08083_08110pd_psiv2.bc 08110_08152pi_psiv2_1sec.bc 08152_08183pe_psiv2_revA.bc 08183_08224pf_psiv2.bc Q8182_08187ra.bc

08187_08192ra.bc 08192_08197ra.bc 08197_08202ra.bc 08202_08207ra.bc 08207_08212ra.bc Q8212 08217ra.bc 08217_08222ra.bc 08224_08257pg_psiv2.bc 08257_08292pe_psiv2.bc 08292_08331ph_fsiv_S45_lud3.bc 08331_09009pf_psiv2.bc
Q9009_09048pi_psiv2.bc 09048_09085ph_psiv2.bc 09048_09085pi_psiv2_B_Branch.bc 09085_09125pe_psiv2.bc 09125_09164pf_fsiv.bc 09164_09204pi_fsiv.bc 09204_09237pg_psiv2.bc 09237_09278pg_fsiv.bc 09278_09317pf_psiv2.bc 09278_09317pg_lud1.bc 09317_09356ph_psiv2.bc 09356_10023pf_psiv2.bc 10023_10060pf_psiv2.bc 10060_10095pf_psiv2.bc 10060_10062pa_lud.bc 10095_10137pf_psiv2.bc 10137_10176pf_psiv2.bc 10176_10211pf_psiv2.bc 10211_10249pf_psiv2.bc 10249_10284pf_psiv2.bc 10284_10328pe_psiv.bc 10328_11017pe_psiv.bc 11017_11066pf_psiv.bc 11066_11115pe_fsiv.bc 11115_11184pd_psiv.bc sat317.bsp or sat351.bsp de421.bsp

\section{References}

Acton, C. H. 1996, Planet. Space Sci., 44, 65

Cooper, N. J., Murray, C. D., Porco, C. C., \& Spitale, J. N. 2006, Icarus, 181, 223

Duriez, L., \& Vienne, A. 1997, A\&A, 324, 366

Eichhorn, H. 1974, Astrometry of star positions (New York: Frederick Ungar)

French, R. G., McGhee, C. A., Frey, M., et al. 2006, PASP, 118, 246

Jacobson, R. A. 1991, A\&A, 90, 541

Jacobson, R. A. 1992, A\&A, 96, 549

Kaplan, G. H., Hughes, J. A., Seidelmann, P. K., \& Smith, C. A. 1989, AJ, 97, 1197

Lainey, V., Karatekin, O., Desmars, J., et al. 2012, ApJ, 752, 14

Mallama, A., Aelion, H. M., \& Mallama, C. A. 2004, Icarus, 167, 320

Noyelles, B., Charnoz, S., Lainey, V., \& Baillié, K. 2012, AAS, DDA meeting, 43, 4.07

Owen Jr., W. M. 2003, Cassini ISS Geometric Calibration of April 2003, JPL IOM 312.E-2003

Peng, Q. Y., Vienne, A., Wu, X. P., Gan, L. L., \& Desmars, J. 2008, AJ, 136, 2214

Porco, C. C., West, R. A., Squyres, S., et al. 2004, Space Sci. Rev., 115, 363 Porco, C. C., Helfenstein, P., Thomas, P. C., et al. 2006, Science, 311, 1393 Stetson, P. B. 1987, PASP, 99, 191

Thomas, P. C., Burns, J. A., Helfenstein, P., et al. 2007, Icarus, 190, 573

Vienne, A., \& Duriez, L. 1995, A\&A, 297, 588

West, R., Knowles, B., Birath, E., et al. 2010, Planet. Space Sci., 58, 1475

Willner, K., Obserst, J., \& Wählisch, M. 2008, A\&A, 488, 361

Zacharias, N., Urban, S. E., Zacharias, M. I., et al. 2004, AJ, 127, 3043 